\title{
Influence of Biochemical and Anthropometric Factors on the Presence of Insulin Resistance in Adolescents
}

Biological Research for Nursing I-8 (C) The Author(s) 2016 Reprints and permission: sagepub.com/journalsPermissions.nav DOI: $10.1177 / 1099800416648207$ brn.sagepub.com

(S)SAGE

\author{
Emilio González-Jiménez, PhD' $^{\prime}$, Jacqueline Schmidt-RioValle, PhD', $^{\prime}$, \\ Miguel A. Montero-Alonso, $\mathbf{P h D}^{2}$, Cristina Padez, $\mathbf{P h D}^{3}$, \\ Carmen J. García-García, $\mathrm{PhD}^{4}$, and Javier S. Perona, $\mathrm{PhD}^{5}$
}

\begin{abstract}
Background: Insulin resistance plays a determinant role in the development of metabolic syndrome in adolescents. The objective of the present study was to determine the influence of factors commonly associated with insulin resistance in a sample of adolescents. Methods: This cross-sectional study included 976 adolescents from southeast Spain. Anthropometric and biochemical measurements were performed, and insulin resistance was assessed using the homeostasis model assessmentinsulin resistance (HOMA-IR). Results: Subjects with abnormal HOMA-IR values had significantly higher body mass index (BMI), body fat content, waist circumference, and systolic blood pressure (BP) than those with normal values. Furthermore, levels of glucose, insulin, glycosylated hemoglobin, total cholesterol, triglycerides, low-density lipoprotein-cholesterol, homocysteine, nonesterified fatty acids, and ceruloplasmin were higher in subjects with abnormal HOMA-IR values. Multivariate logistic regression analysis showed the highest odds ratio $(O R)$ for $\mathrm{BMI}$ and that combinations of BMI with body fat content or systolic BP can increase the risk of insulin resistance 7-fold. Discussion: Anthropometric indicators have different levels of influence on the risk of insulin resistance in adolescents, and a combination of two of these indicators is enough to increase the risk 7-fold. Since the highest OR was observed for BMI, the greatest effort should be directed to reducing this parameter in adolescents. An adequate understanding by nursing personnel of factors associated with insulin resistance is a key factor in the prevention of this pathophysiological condition and its complications in adolescents.
\end{abstract}

\section{Keywords}

insulin resistance, risk factors, metabolic syndrome, HOMA-IR, adolescence

Insulin resistance is a multifactorial process that is established when the amounts of insulin produced physiologically lose the ability to regulate the metabolism of carbohydrates, lipids, and proteins. In general, insulin resistance comprises a decrease in glucose transport induced by the hormone in adipocytes and skeletal muscle, an increase in hepatic glucose output, and alterations in lipid metabolism in adipose and liver tissues (Aguilar Cordero et al., 2010).

The worldwide proliferation of obesity in the adolescent population is responsible for a corresponding increase in insulin resistance among young people in the developed countries (Chung et al., 2013; Lin et al., 2015). The excess energy associated with obesity may result in hyperplasia and hypertrophy of adipocytes, leading to oxidative stress. This oxidative stress of adipocytes induces a chronic low-level inflammation in adipose tissue and the production of adipokines, nonesterified fatty acids (NEFA), and inflammatory mediators. This inflammation, in turn, is related to insulin resistance and impaired insulin secretion by the pancreatic $\beta$ cells. Finally, this process causes dysregulation of glucose homeostasis and development of type 2 diabetes mellitus (T2DM; Van der Aa, Fazeli Farsani, Knibbe, De Boer, \& Van der Vorst, 2015). In addition, studies have suggested that subjects with abnormal homeostasis model assessment-insulin resistance (HOMA-IR) values often show elevated levels of homocysteine, NEFA, and ceruloplasmin, which might constitute an additional risk factor for the premature development of cardiovascular pathology (Zulet, Puchau, Navarro, Marti, \& Martinez, 2007).

\footnotetext{
' Department of Nursing, University of Granada, Granada, Spain

${ }^{2}$ Department of Statistics and Operations Research, University of Granada, Melilla, Spain

${ }^{3}$ Department of Life Sciences, University of Coimbra, Coimbra, Portugal

${ }^{4}$ Department of Forensic Medicine, Toxicology and Physical Anthropology, University of Granada, Granada, Spain

${ }^{5}$ Department of Food and Health, Instituto de la Grasa (CSIC), Seville, Spain
}

\section{Corresponding Author:}

Javier S. Perona, PhD, Department of Food and Health, Instituto de la GrasaCSIC, Campus Universidad Pablo de Olavide, Edificio 46, 4I0I3, Seville, Spain. Email: perona@ig.csic.es 
It is a widely accepted hypothesis that obesity and insulin resistance are the main factors in the etiology of the metabolic syndrome (MetS), a group of risk factors that, when occurring together, increase the risk of cardiovascular disease and T2DM (Cerezo, Segura, Praga, \& Ruilope, 2013).

Studies carried out to date have used differing criteria to define the MetS in adolescents (Cook, Weitzman, Auinger, Nguyen, \& Dietz, 2003; Cruz et al., 2004; De Ferranti et al., 2004; McGillis Bindler, Massey, Shultz, Mills, \& Short, 2007; Weiss et al., 2004). In 2007, the International Diabetes Federation published a set of guidelines for the diagnosis of MetS in children and adolescents that established a simple unified definition (Zimmet et al., 2007). According to this definition, the identification of MetS in adolescents is based on a waist circumference (WC) of $94 \mathrm{~cm}$ or more in males and $80 \mathrm{~cm}$ or more in females in combination with other risk factors, such as fasting glucose levels of $100-125 \mathrm{mg} / \mathrm{dl}$, triglyceride levels of least $150 \mathrm{mg} / \mathrm{dl}$, and high-density lipoprotein (HDL)-cholesterol levels lower than $40 \mathrm{mg} / \mathrm{dl}$ in males and $50 \mathrm{mg} / \mathrm{dl}$ in females, along with a blood pressure (BP) of $130 / 85 \mathrm{mmHg}$.

Mills et al. (2004) studied a population of 1,865 children and adolescents, 6-18 years of age, over a period of 6 years and showed that insulin resistance was more frequent in those children who subsequently developed MetS, which seems to indicate that insulin resistance precedes the appearance of MetS in childhood. Cruz et al. (2004) analyzed the role of insulin resistance in the development of MetS in obese children of Hispanic descent and reported that sensitivity to insulin was $62 \%$ lower among those who had MetS. Furthermore, the authors concluded that insulin sensitivity was independently and negatively related to triglyceride concentrations and BP levels and positively related to levels of HDL-C. These results suggest that the effects of adiposity on dyslipidemia and BP are mediated by insulin resistance.

Taken together, these findings seem to indicate that an initial state of obesity and subsequent insulin resistance both have an important role in the development of MetS. Nevertheless, in obese subjects, insulin resistance seems to play a larger role in the development of MetS than the degree of obesity (Rodrigues, Abreu, Resende, Goncalves, \& Gouvea, 2013). Researchers have generally assumed that intra-abdominal (or visceral) fat accumulation is a phenomenon associated mainly with adulthood. However, subcutaneous adiposity at the waist is a more significant predictor of MetS traits in children and adolescents than it is in adults (Ali et al., 2014). As a consequence, an increasing number of studies are showing that $\mathrm{WC}$ is the best measure for identifying children with insulin resistance and hence those most at risk for MetS (Esmaillzadeh, Mirmiran, Azadbakht, Amiri, \& Azizi, 2006; Hirschler, Aranda, Calcagno, Maccalini, \& Jadzinsky, 2005; Moreno et al., 2002).Yet, it remains to be definitively demonstrated whether WC measurement alone is sufficient to identify those children at greatest risk for obesity-related insulin resistance.

Therefore, the purpose of this study was to determine the level of influence of body mass index (BMI), WC, body fat content, and $\mathrm{BP}$ on the development of insulin resistance in adolescents in order to establish which is the most prominent. Findings will help researchers and health-care professionals to develop preventive strategies that reduce adolescents' risk of developing MetS.

\section{Method}

\section{Design and Setting}

We carried out this cross-sectional study in the province of Granada in southeast Spain. To obtain a representative sample, we performed a demographic analysis in order to characterize the population of adolescents between the ages of 10 and 15 . According to the municipal census of 2008, the population of adolescents of this age was 49,359 , of which 24,055 (48.7\%) were boys and 25,304 (51.3\%) were girls. Based on these data and assuming an error of $3 \%$, we decided that our sample size would be 976 adolescents (519 females and 457 males), 10-15 years of age, all of Spanish origin, drawn from the sixth grade of primary school to the third year of secondary school. The subjects attended 18 schools in the province of Granada. Among the 18 selected schools, we randomly selected two classes per grade from which to invite participants. The participants had similar socioeconomic statuses.

The Board of Education of the Andalusian Regional Government (Granada Delegation) approved the study, and the school directors authorized it as well. The Ethics Committee of the University of Granada (EC-47851) also approved the study, including the model of informed consent. Parents or guardians explicitly authorized their children's participation in the study by written informed consent. E.G.-J., member of the research team, recorded and protected informed consents, and they remain under his custody. An important condition for inclusion was that participants had to be healthy and not have any type of endocrine dysfunction, physical disorder, or infectious process. We performed this research in strict compliance with the international code of medical ethics established by the World Medical Association and the Declaration of Helsinki.

\section{Measures}

Anthropometric measurements. Each participant underwent a complete anthropometric evaluation performed according to the recommendations of the European Pediatric Association (Body Composition Analysis Protocol). The variables assessed were weight, height, and BMI. We performed the anthropometric measurements in the morning after a 12-hr fast and a 24-hr abstention from exercise. We measured body weight $(\mathrm{kg})$ twice (with participants wearing no shoes and in light clothes) using a self-calibrating Seca ${ }^{\circledR} 861$ class (III) digital floor scale (Saint Paul, USA) with a precision of up to $100 \mathrm{~g}$. We measured height with a Seca $214^{*}$ portable stadiometer, asking participants to remove their shoes and stand erect with their backs, buttocks, and heels in continuous contact with the vertical height rod of the stadiometer and head oriented in the Frankfurt plane. We then placed the horizontal headpiece on top of the participants' heads to measure their height. We took height 
measurements twice to the nearest $0.5 \mathrm{~cm}$. We used the average of the two values for weight and height in the analysis. We calculated BMI as weight divided by height squared $\left(\mathrm{kg} / \mathrm{m}^{2}\right)$. The same trained research assistant performed all the measurements. We defined overweight and obesity, according to the international standards established by Cole, Bellizzi, Flegal, and Dietz (2000), as values above the 85th and 95th percentiles, respectively, for BMI for age and sex. We also evaluated the triceps, biceps, subscapular, and suprailiac skinfolds with a Holtain ${ }^{\circledR}$ skinfold caliper (Saint Paul, USA), which has a precision of $0.1-0.2 \mathrm{~mm}$ and used these measurements to calculate the percentage of body fat. The first step in determining percentage of body fat was to calculate body density using Brook's equation (Brook, 1971):

$$
\begin{aligned}
\text { Boys : density }= & 1.1690-0.0788 \times \log 10 \text { [triceps } \\
& + \text { biceps }+ \text { subscapular }+ \text { suprailiac]. } \\
\text { Girls : density }= & 1.2063-0.0999 \times \log 10 \text { [triceps } \\
& + \text { biceps }+ \text { subscapular }+ \text { suprailiac]. }
\end{aligned}
$$

After determining body density, we used Siri's (1961) equation to calculate body fat percentage: body fat percentage $=$ $[(4.95 /$ density $)-4.5] \times 100$. We measured the WC of participants. We measured WC with a Seca automatic roll-up measuring tape (precision of $1 \mathrm{~mm}$ ) using the horizontal plane midway between the lowest rib and the upper border of the iliac crest at the end of a normal inspiration/expiration. We measured hip circumference at the maximum extension of the buttocks as viewed from the right side.

$B P$ determination. We measured $\mathrm{BP}$ levels using a previously calibrated aneroid sphygmomanometer and a Littmann ${ }^{\circledR}$ stethoscope (Saint Paul, USA). We took all measurements using the right arm after each participant had rested for at least $15 \mathrm{~min}$ in a sitting position. We recorded the average of two readings obtained at a minimum of 5 min apart. Systolic BP was based on Korotkoff Phase I and diastolic BP was based on Phase V. Systolic BP $\geq 130$ and/or diastolic BP $\geq 85 \mathrm{mmHg}$ were regarded as a risk factor of MetS.

\section{Serum Biochemical Analysis}

At 8:00 a.m. after participants had fasted for $12 \mathrm{hr}$ overnight, we extracted $10 \mathrm{ml}$ of blood by venipuncture in the antecubital fossa of the right arm with a disposable vacuum blood collection tube. Within $4 \mathrm{hr}$ after the extraction, we centrifuged the samples at 3,500 rpm for $15 \mathrm{~min}$ (Z400 K, Hermle, Wehingen, Germany) to separate the red blood cells. We froze the serum at $-80^{\circ} \mathrm{C}$ for subsequent analysis.

Immediately after collection and before centrifugation, however, we measured glucose concentration using an enzymatic colorimetric method (glucose oxidase-phenolaminophenazone [GOD-PAP] method, Human Diagnostics, Germany) as well as the concentrations of HDL-C, total cholesterol, and triglycerides by means of enzymatic colorimetric methods using an Olympus analyzer. Low-density lipoprotein-cholesterol (LDL-C) was estimated using the Friedewald equation ( $[\mathrm{LDL}-\mathrm{C}]=[$ Total cholesterol $]-$ $[\mathrm{HDL}-\mathrm{C}]-([\mathrm{TG}] / 5))$, where $\mathrm{TG}=$ concentration of triglycerides. The remaining analyses were conducted using the samples that had been frozen. We determined serum levels of homocysteine $(\mu \mathrm{mol} / \mathrm{L})$ with the enzymatic assay. For the determination of ceruloplasmin levels, however, we used an immunoturbidometric assay (Architect ci8200, Abbott, Abbott Park, IL), which had an intra-assay variation coefficient of $3.7 \%$, an inter-assay precision of up to $4 \%$, and a reference of $20-60 \mathrm{mg} / \mathrm{dl}$. Serum insulin was determined by radioimmunoanalysis (Insulin Kit, DPC, Los Angeles, CA). We measured the glycosylated hemoglobin ( $\mathrm{HbA} 1 \mathrm{c})$ by high-performance liquid chromatography using an automated analyzer certified by the National Glycohemoglobin Standardization Program (model HLC-723 G7, Tosoh, Tokyo, Japan; intra-assay coefficient of variation $<0.8 \%$, interassay coefficient of variation $<0.5 \%$ ) and standardized the levels according to the Diabetes Control and Complications Trial.

We quantified insulin resistance (IR) using HOMA (Matthews et al., 1985) by applying the following formula: fasting glucose $(\mathrm{mmol} / \mathrm{L}) \times$ fasting insulin $(\mathrm{mU} / \mathrm{L}) / 22.5$. We divided participants into two groups based on their HOMA-IR, calculating the HOMA-IR cutoff point, using its receiver operating characteristic curve, as 3.83 with a sensitivity of $80.5 \%$ and a specificity of $79.8 \%$. Therefore, HOMA-IR $>3.83$ was regarded as abnormal and HOMA-IR $<3.83$ as normal.

\section{Statistical Analysis}

We compared the mean values of continuous variables using Student's $t$-test and the categorical variables between abnormal and normal HOMA-IR groups using $\chi^{2}$. We assessed the level of influence of BMI, body fat percentage, WC, and systolic BP on IR by calculating the odds ratio $(O R)$ and the $95 \%$ confidence intervals (CIs) for the abnormal HOMA-IR group in comparison to the normal group. We used a multivariate logistic regression model for this purpose because we found these factors to be significant in the univariate model. After adjusting the risk factors, we combined the effects of the BMI, WC, and systolic BP to examine the combined risk effect for HOMA-IR abnormality. Two-sided $p$ values $<.05$ were regarded as statistically significant. We analyzed data using SPSS software for Linux 155, Version 21.0 (IBM Corp, Armonk, NY, USA).

\section{Results}

Table 1 lists the physical characteristics of the participants, who were divided into two groups: (i) $28.1 \%$ had abnormal HOMA-IR values (> 3.83) and (ii) $71.9 \%$ had normal HOMA-IR values $(<3.83)$. BMI, body fat percentage, WC, and systolic and diastolic BP values were significantly higher in participants with abnormal HOMA-IR values compared to those with normal values $(p<.001)$. However, we found no significant correlation between HOMA-IR group and age $(p=.202)$ or gender $(p=.510)$. 
Table I. Physical Characteristics of Participants by HOMA-IR Group.

\begin{tabular}{lccc}
\hline Variable & $\begin{array}{c}\text { Abnormal } \\
\text { HOMA-IR } \\
(n=274)\end{array}$ & $\begin{array}{c}\text { Normal } \\
\text { HOMA-IR } \\
(n=702)\end{array}$ & $p$ \\
\hline Age (years) & $13.2 \pm \mathrm{I}$.I & $13.1 \pm \mathrm{I} . \mathrm{I}$ & $.202^{\mathrm{a}}$ \\
Gender (male) & $14 \mathrm{I}(5 \mathrm{I} .4)$ & $316(45.0)$ & $.510^{\mathrm{b}}$ \\
Body mass index $\left(\mathrm{kg} / \mathrm{m}^{2}\right)$ & $24.9 \pm 4.1$ & $19.8 \pm 2.5$ & $<.00 \mathrm{I}^{\mathrm{a}}$ \\
Body fat $(\%)$ & $35.0 \pm 7.5$ & $26.0 \pm 6.8$ & $<.00 \mathrm{I}^{\mathrm{a}}$ \\
Waist circumference $(\mathrm{cm})$ & $8 \mathrm{I} .7 \pm 12.0$ & $68.6 \pm 7.7$ & $<.00 \mathrm{I}^{\mathrm{a}}$ \\
Systolic BP $(\mathrm{mmHg})$ & $127.2 \pm 16.8$ & $114.4 \pm 13.3$ & $<.00 \mathrm{I}^{\mathrm{a}}$ \\
Diastolic BP $(\mathrm{mmHg})$ & $68.1 \pm 9.1$ & $62.5 \pm 8.4$ & $<.00 \mathrm{I}^{\mathrm{a}}$ \\
\hline
\end{tabular}

Note. All values are expressed as means $\pm S D$ s or $n(\%)$. BP $=$ blood pressure; HOMA-IR $=$ homeostasis model assessment for insulin resistance.

${ }^{\text {a }}$ Student's t-test. ${ }^{\mathrm{b}} \chi^{2}$ test.

Table 2. Serum Biomarkers for Participants by HOMA-IR Group.

\begin{tabular}{|c|c|c|c|}
\hline Variables & $\begin{array}{l}\text { Abnormal } \\
\text { HOMA-IR } \\
(n=274)\end{array}$ & $\begin{array}{c}\text { Normal } \\
\text { HOMA-IR } \\
(n=702)\end{array}$ & $p^{a}$ \\
\hline Glucose level (mmol/L) & $6.1 \pm 2.7$ & $4.3 \pm 0.3$ & $<.001$ \\
\hline Insulin (mU/L) & $29.5 \pm 14.5$ & $17.0 \pm 1.1$ & $<.001$ \\
\hline HBAIc (\%) & $5.9 \pm 3.1$ & $4.2 \pm 0.3$ & $<.001$ \\
\hline Total cholesterol (mg/dl) & $91.9 \pm 23.2$ & $77.5 \pm 10.3$ & $<.001$ \\
\hline Triglycerides (mg/dl) & $155.8 \pm 93.6$ & $1 \mid 5.7 \pm 4.0$ & $<.001$ \\
\hline HDL-C (mg/dl) & $38.0 \pm 3.7$ & $40.9 \pm 2.1$ & $<.001$ \\
\hline LDL-C (mg/dl) & $112.4 \pm 35.0$ & $85.7 \pm 8.1$ & $<.001$ \\
\hline Homocysteine $(\mu \mathrm{mol} / \mathrm{L})$ & $12.2 \pm 4.4$ & $8.0 \pm 2.8$ & $<.001$ \\
\hline NEFA (mmol/L) & $0.36 \pm 0.22$ & $0.19 \pm 0.06$ & $<.001$ \\
\hline Ceruloplasmin (mg/dl) & $42.8 \pm 26.4$ & $21.3 \pm 2.0$ & $<.001$ \\
\hline
\end{tabular}

Note. All values are expressed as means $\pm S D s . H B A I c=$ glycosylated hemoglobin; HDL-C = high-density lipoprotein cholesterol; HOMA-IR = homeostasis model assessment for insulin resistance; LDL-C = low-density lipoprotein cholesterol; NEFA = nonesterified fatty acids.

${ }^{\text {aS }}$ tudent's $t$-test.

Table 2 shows the serum biochemical data for the 976 participants with either normal or abnormal HOMA-IR values. The levels of glucose, insulin, HBA1c, total cholesterol, triglycerides, LDL-C, homocysteine, NEFA, and ceruloplasmin were significantly higher $(p<.001)$ in participants with abnormal HOMA-IR values. It is noteworthy that, besides the more common parameters such as glucose, insulin, HBA1c, triglycerides, NEFA, total cholesterol, LDL-C, and HDL-C, less common parameters like homocysteine and ceruloplasmin differed between the two groups as well.

As shown in Table 3, the crude ORs of HOMA-IR abnormality were $38.71,6.89,9.27$, and 4.78, respectively, for BMI, body fat content, WC, and systolic BP, with all of them showing statistical significance. After adjusting for gender, we found that the $O R$ s were similar to the crude $O R$ values. Likewise, after adjustment for gender, cholesterol, triglycerides, HDL-C, and LDL-C, the multivariate $O R$ of HOMA-IR abnormality was notably higher for BMI than for body fat content, WC, and systolic BP.

The combined effect on the risk of abnormal HOMA-IR among three risk factors - body fat content, BMI, and systolic
$\mathrm{BP}$ - is shown in Table 4. Compared with a reference group that included subjects without any of these three risk factors, those with all risk factors had a significantly higher risk of abnormal HOMA-IR $(O R=101.45 ; 95 \% \mathrm{CI}=[43.02,239.24])$. Furthermore, participants with one or any combination of two of these three factors had 14.86- or 55.30-fold increased risk for HOMAIR abnormality over the reference group, respectively. The $O R$ was unaffected by gender. This finding shows a significant combined effect on risk of HOMA-IR abnormality for WC, BMI, and systolic BP among adolescents.

\section{Discussion}

Several studies have shown that a transient physiological insulin resistance develops in the pubertal period, although its cause is not fully understood (Kurtoğlu et al., 2010). Nevertheless, researchers have widely accepted the conclusion that obesity is responsible for the recent increases in the prevalence of insulin resistance in children and adults (Cruz et al., 2004; Mills et al., 2004; Van der Aa et al., 2015). In this regard, there has been an increase in maturity-onset diabetes in obese youth and, because of the transient physiological status of insulin resistance, there is extra stress on the $\beta$ cells in these youth. Among the obesityrelated factors associated with insulin resistance, $\mathrm{WC}$ has been one of the most interesting for diagnostic purposes in children (Esmaillzadeh et al., 2006; Hirschler et al., 2005; Moreno et al., 2002). However, there is still debate as to whether WC alone can be used to identify adolescents with insulin resistance. Therefore, in the present study, we aimed to determine the level of influence of obesity-related factors on the risk for insulin resistance in order to establish which has the greatest effect in adolescents and to ascertain if a single factor could be used to identify insulin resistance in this population.

Our results reinforce the findings of previous studies (Lin et al., 2015; Reaven, 2004; Rogero Blanco et al., 2012) and are novel in the context of the Spanish population, which furthers the argument for generalizability of the cause of IR. Our findings regarding the individual and collective influence of the factors involved in the development of insulin resistance in our young sample provide information about the individual and collective usefulness of these parameters for assessing the risk of developing MetS. These findings are particularly important because in most other studies on this topic, the sample population comprised adults (Rogero Blanco et al., 2012).

Our results show that there is a positive association between abnormal HOMA-IR values and the biological/physiological indicators of BMI, body fat content, WC, and systolic BP. As Lin et al. (2015) also highlighted, the close association between these anthropometric variables and BP levels in relation to abnormal HOMA-IR values (i.e., their combined effect) indicates that this relationship should be regarded as a predictive risk factor for insulin resistance. Similar to Reaven's (2004) study, our results show the effects of BMI, body fat percentage, $\mathrm{WC}$, and systolic BP levels, factors that heighten the risk of an abnormal HOMA-IR. In the present study, BMI had the highest predictive value, as assessed by using the $O R$ of HOMA-IR as a 
Table 3. Multivariate Odds Ratios (ORs) of HOMA-IR Abnormality by Body Mass Index (BMI), Body Fat, Waist Circumference, and Systolic Blood Pressure.

\begin{tabular}{|c|c|c|c|c|c|}
\hline Variable & \multicolumn{2}{|c|}{ HOMA-IR } & Crude $O R(\mathrm{Cl})$ & $O R^{\mathrm{a}}(\mathrm{Cl})$ & $O R^{b}(\mathrm{Cl})$ \\
\hline Normal & 144 & 686 & 1.00 & 1.00 & 1.00 \\
\hline Abnormal & 130 & 16 & $38.71[22.34,67.05]^{*}$ & $38.61[22.28,66.92]^{*}$ & I58.37 [78.46, 319.67$]^{*}$ \\
\hline \multicolumn{6}{|l|}{ Body fat } \\
\hline \multicolumn{6}{|l|}{ WC } \\
\hline Normal & 61 & 510 & 1.00 & 1.00 & 1.00 \\
\hline Abnormal & 213 & 192 & $9.27[6.67,12.89]^{*}$ & $9.23[6.63,12.86]^{*}$ & $45.46[23.97,86.23]^{*}$ \\
\hline \multicolumn{6}{|l|}{ Systolic BP } \\
\hline Normal & 102 & 519 & 1.00 & 1.00 & 1.00 \\
\hline Abnormal & 172 & 183 & $4.78[3.55,6.44]^{*}$ & $4.74[3.52,6.39]^{*}$ & $7.94[5.40,11.66]^{*}$ \\
\hline
\end{tabular}

Note. $\mathrm{BP}=$ blood pressure; $\mathrm{Cl}=$ confidence interval; HOMA-IR = homeostasis model assessment for insulin resistance; WC $=$ waist circumference. ${ }^{\mathrm{a} O R}$ adjusted for gender. ${ }^{\mathrm{b}} \mathrm{OR}$ adjusted for gender, cholesterol, triglycerides, high- and low-density lipoprotein cholesterol. $*_{p}<.001$.

Table 4. Combined Effects of Body Fat, Body Mass Index (BMI), and Systolic Blood Pressure (SBP) on Risk of HOMA-IR Abnormality.

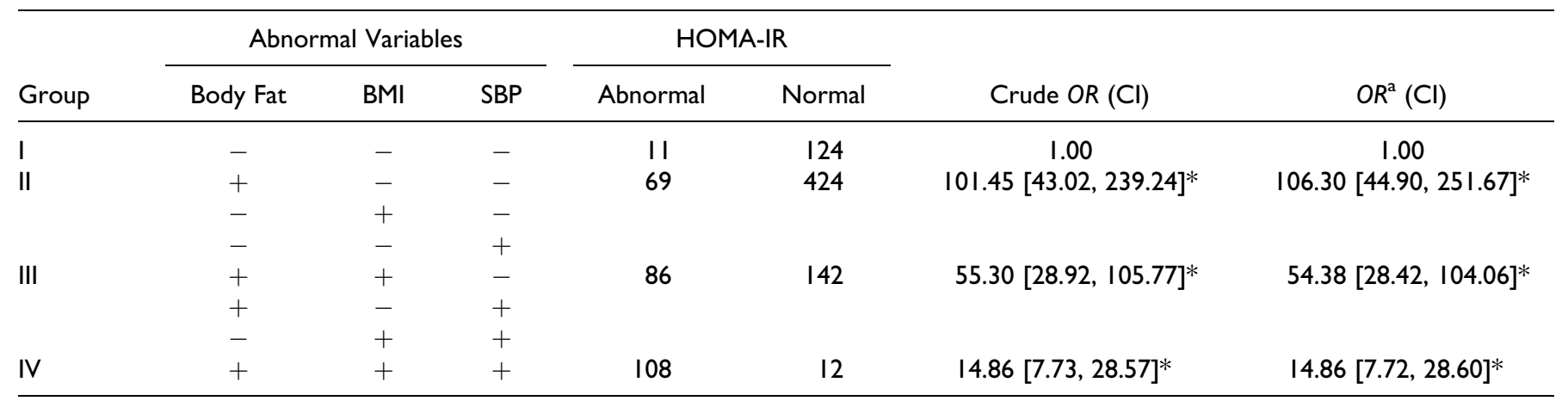

Note. Plus signs indicate an abnormal level of a variable, while minus signs indicate a normal level. HOMA-IR $=$ homeostasis model assessment for insulin resistance; $O R=$ odds ratio; $\mathrm{SBP}=$ systolic blood pressure.

${ }^{a} O R$ adjusted for gender.

$*_{p}<.001$.

determiner compared to $\mathrm{WC}$, body fat (\%), and systolic BP. The $O R$ value was highly increased, as we adjusted it for gender and levels of cholesterol, triglycerides, HDL-C, and LDL-C.

Our biochemical study revealed that participating youth with an abnormal HOMA-IR level had elevated levels of almost all of the parameters we studied. However, we found no significant correlation between gender and HOMA-IR group (abnormal vs. normal), which was unexpected because previous authors have proposed that girls are more resistant to insulin compared to boys. In a systematic review, Van der Aa, Fazeli Farsani, Knibbe, De Boer, and Van der Vorst (2015) found that 7 of the 13 studies reported sex-specific prevalence rates of insulin resistance. However, the authors pointed out that the reason for this sex difference is unknown because it was not associated with either the pubertal state or adipose tissue content.

High levels of homocysteine, NEFA, and ceruloplasmin are additional risk factors for insulin resistance and for the early development of cardiovascular disorders such as arteriosclerosis, coronary artery blockage, and high BP, as described in previous research (Fridman, D' Eramo, \& Finkelstein, 1997; Zulet et al., 2007). Others have pointed out that homocysteine levels are not a relevant cardiovascular risk factor in adolescents that consume adequate diets (Bindler, Massey, Shultz, Mills, \& Short, 2004). Bindler et al.'s results are in agreement with those of Papandreou, Mavromichalis, Makedou, Rousso, and Arvanitidou (2006), who explored a similar question in a sample of 524 Greek adolescents. The disparity between the traditional concerns regarding homocysteine and these new observations justifies the continued investigation of the potential influence of homocysteine on cardiovascular risk, especially when there are insufficient reference data in representative samples of adolescent populations (Must, Jacques, Rogers, Rosenberg, \& Selhub, 2003). Likewise, controversy remains regarding the clinical significance of NEFA and ceruloplasmin levels and not only in adolescents. There is 
widespread acceptance that NEFA can mediate insulin resistance via their release by adipose tissue. However, Karpe, Dickmann, and Frayn (2011) reexamined this concept on the basis of newly available literature and concluded that more emphasis should be placed on alternative explanations for the relationship between obesity and insulin resistance, such as impaired adipose tissue fat storage or dysfunctional regulation of adipokines or adipose-related inflammatory cytokines.

We believe that ceruloplasmin should be considered a promising marker for insulin resistance. Ceruloplasmin is released by the liver and is the main copper-carrying protein; as an acute-phase protein, it is also related to systemic inflammation. The associations between ceruloplasmin and factors related to insulin resistance are still controversial. Although researchers have found ceruloplasmin to be independently associated with intra-abdominal fat thickness (Cignarelli et al., 1996) and elevated in subjects with MetS (Kim et al., 2002), others did not find any kind of association between ceruloplasmin and obesity (Safavi, Ziaei, \& Maracy, 2012). In Spanish adolescents, Wärnberg et al. (2006) found significant correlations between BMI and $\mathrm{WC}$ with ceruloplasmin, and, more recently, we reported significant correlations between ceruloplasmin and BMI $z$-score in nonobese and obese subjects (Aguilar, GonzálezJiménez, Antelo, \& Perona, 2013). While our data are in agreement with those of some of these authors, it is obvious that there are contradictions that justify the inclusion of these variables in future studies.

Furthermore, the high levels of insulin, HBA1c, total cholesterol, triglycerides, and LDL cholesterol in those youth with abnormal HOMA-IR values in the present study were in close agreement with the results previous researchers have obtained (Park et al., 2012; Pastucha et al., 2013). Nonetheless, the crosssectional nature of this study limits our ability to detect causal relationships between each of the factors analyzed and the possible development of insulin resistance. In this sense, and as Lin et al. (2015) underlined, future studies should focus on the monitoring and evaluation over time of all the potentially influential factors and circumstances in the genesis of MetS at an early age.

In summary, our findings confirmed anthropometric indicators such as BMI, body fat content, and WC as well as other parameters such as systolic BP and all of the biochemical markers we analyzed as important risk factors for the development of insulin resistance. We found that BMI exerts the greatest influence and that the contributions of any two factors combined are enough to raise the risk 7-fold in terms of $O R$. These markers could also play an important role in later development of MetS, T2DM, and cardiovascular diseases. Nurses could, thus, use simple anthropometric measures and biochemical analyses to identify children and adolescents at risk of developing insulin resistance, which could be extremely useful for preventing diseases related to MetS in adulthood.

\section{Acknowledgments}

We are grateful to the participating schools, parents, and guardians as well as to the students for their collaboration in the development of this study.

\section{Author Contribution}

Emilio González-Jiménez contributed to design, acquisition, and analysis; critically revised the manuscript; gave final approval; and agrees to be accountable for all aspects of work ensuring integrity and accuracy. Jacqueline Schmidt-RioValle contributed to analysis, critically revised the manuscript, gave final approval, and agrees to be accountable for all aspects of work ensuring integrity and accuracy. Miguel A. Montero-Alonso contributed to analysis and interpretation, critically revised the manuscript, gave final approval, and agrees to be accountable for all aspects of work ensuring integrity and accuracy. Carmen J. García-García contributed to conception, critically revised the manuscript, gave final approval, and agrees to be accountable for all aspects of work ensuring integrity and accuracy. Cristina Padez contributed to acquisition, critically revised the manuscript, gave final approval, and agrees to be accountable for all aspects of work ensuring integrity and accuracy. Javier S. Perona contributed to design, analysis, and interpretation; critically revised the manuscript; gave final approval; and agrees to be accountable for all aspects of work ensuring integrity and accuracy.

\section{Declaration of Conflicting Interests}

The author(s) declared no potential conflicts of interest with respect to the research, authorship, and/or publication of this article.

\section{Funding}

The author(s) disclosed receipt of the following financial support for the research, authorship, and/or publication of this article: This work was supported by funds from the Spanish Ministry of Economy and Competitiveness (AGL2011-23810).

\section{References}

Aguilar, M. J., González-Jiménez, E., Antelo, A., \& Perona, J. S. (2013). Insulin resistance and inflammation markers: Correlations in obese adolescents. Journal of Clinical Nursing, 22, 2002-2010.

Aguilar Cordero, M. J., González Jiménez, E., Sánchez Perona, J., Padilla López, C. A., Álvarez Ferré, J., Mur Villar, N., \& Rivas García, F. (2010). The Guadix study of the effects of a Mediterranean-diet breakfast on the postprandial lipid parameters of overweight and obese pre-adolescents. Nutrición Hospitalaria, $25,1025-1033$.

Ali, O., Cerjak, D., Kent, J. W. J., James, R., Blangero, J., \& Zhang, Y. (2014). Obesity, central adiposity and cardiometabolic risk factors in children and adolescents: A family-based study. Pediatric Obesity, 9, e58-e62.

Bindler, R. C., Massey, L. K., Shultz, J. A., Mills, P. E., \& Short, R. (2004). Homocysteine in a multi-ethnic sample of school-age children. Journal of Pediatric Endocrinology and Metabolism, 17, 327-337.

Brook, C. G. D. (1971). Determination of body composition of children from skinfold measurements. Archives of Disease in Childhood, 46, 182-184.

Cerezo, C., Segura, J., Praga, M., \& Ruilope, L. M. (2013). Guidelines updates in the treatment of obesity or metabolic syndrome and hypertension. Current Hypertension Reports, 15, 196-203.

Chung, J. Y., Kang, H. T., Shin, Y. H., Lee, H. R., Park, B. J., \& Lee, Y. J. (2013). Prevalence of metabolic syndrome in children and 
adolescents - The recent trends in South Korea. Journal of Pediatric Endocrinology and Metabolism, 26, 105-110.

Cignarelli, M., DePergola, G., Picca, G., Sciaraffia, M., Pannacciulli, N., Tarallo, M., ... Giorgino, R. (1996). Relationship of obesity and body fat distribution with ceruloplasmin serum levels. International Journal of Obesity and Related Metabolic Disorders, 20, 809-813.

Cole, T. J., Bellizzi, M. C., Flegal, K. M., \& Dietz, W. H. (2000). Establishing a standard definition for child overweight and obesity worldwide: International survey. British Medical Journal, 320, 1240-1243.

Cook, S., Weitzman, M., Auinger, P., Nguyen, M., \& Dietz, W. H. (2003). Prevalence of a metabolic syndrome phenotype in adolescents: Findings from the third National Health and Nutrition Examination Survey, 1988-1994. Archives of Pediatrics \& Adolescent Medicine, 157, 821-827.

Cruz, M., Weigensberg, M., Huang, T., Ball, G., Shaibi, G., \& Goran, M. (2004). The metabolic syndrome in overweight Hispanic youth and the role of insulin sensitivity. Journal of Clinical Endocrinology \& Metabolism, 89, 108-113.

De Ferranti, S. D., Gauvreau, K., Ludwig, D. S., Neufeld, E. J., Newburger, J. W., \& Rifai, N. (2004). Prevalence of the metabolic syndrome in American adolescents: Findings from the third National Health and Nutrition Examination Survey. Circulation, 110, 2494-2497.

Esmaillzadeh, A., Mirmiran, P., Azadbakht, L., Amiri, P., \& Azizi, F. (2006). Independent and inverse association of hip circumference with metabolic risk factors in Tehranian adult men. Preventive Medicine, 42, 354-357.

Fridman, O., D’ Eramo, J. L., \& Finkelstein, A. E. (1997). Homocisteina plasmatica: Factor de riesgo independiente de afecciones vasculares oclusivas. Revista Argentina de Cardiología, 65, $571-581$

Hirschler, V., Aranda, C., Calcagno, M. L., Maccalini, G., \& Jadzinsky, M. (2005). Can waist circumference identify children with the metabolic syndrome? Archives of Pediatrics \& Adolescent Medicine, 159, 740-744.

Karpe, F., Dickmann, J. R., \& Frayn, K. N. (2011). Fatty acids, obesity, and insulin resistance: Time for a reevaluation. Diabetes, 60 , 2441-2449.

Kim, C. H., Park, J. Y., Kim, J. Y., Choi, C. S., Kim, Y. I., Chung, Y. E., ... Lee, K. U. (2002). Elevated serum ceruloplasmin levels in subjects with metabolic syndrome: A population-based study. Metabolism, 51, 838-842.

Kurtoğlu, S., Hatipoğlu, N., Mazıcıŏglu, M., Kendirici, M., Keskin, M., \& Kondolot, M. (2010). Insulin resistance in obese children and adolescents: HOMA-IR cut-off levels in the prepubertal and pubertal periods. Journal of Clinical Research in Pediatric Endocrinology, 2, 100-106.

Lin, S. Y., Su, C. T., Hsieh, Y. C., Li, Y. L., Chen, Y. R., Cheng, S. Y., \& Chiou, H. Y. (2015). Risk factors correlated with risk of insulin resistance using homeostasis model assessment in adolescents in Taiwan. Asia-Pacific Journal of Public Health, 27, 476-484.

Matthews, D., Hosker, J., Rudenski, A., Naylor, B., Treacher, D., \& Turner, R. (1985). Homeostasis model assessment: Insulin resistance and B-cell function from fasting plasma glucose and insulin concentrations in man. Diabetologia, 28, 412-419.

McGillis Bindler, R. C., Massey, L. K., Shultz, J. A., Mills, P. E., \& Short, R. (2007). Metabolic syndrome in a multiethnic sample of school children: Implications for the pediatric nurse. Journal of Pediatric Nursing, 22, 43-58.

Mills, G. W., Avery, P., McCarthy, M., Hattersley, A. T., Levy, J. C., Hitman, G. A., ... Walker, M. (2004). Heritability estimates for beta cell function and features of the insulin resistance syndrome in UK families with an increased susceptibility to Type 2 diabetes. Diabetologia, 47, 732-738.

Moreno, L. A., Pineda, I., Rodríguez, G., Fleta, J., Sarría, A., \& Bueno, M. (2002). Waist circumference for the screening of the metabolic syndrome in children. Acta Paediatrica, 91, $1307-1312$.

Must, A., Jacques, P. F., Rogers, G., Rosenberg, I. H., \& Selhub, J. (2003). Serum total homocysteine concentrations in children and adolescents: Results from the third National Health and Nutrition Examination Survey (NHANES III). Journal of Nutrition, 133, 2643-2649.

Papandreou, D., Mavromichalis, I., Makedou, A., Rousso, I., \& Arvanitidou, M. (2006). Total serum homocysteine, folate and vitamin B12 in a Greek school age population. Clinical Nutrition, 25, 797-802.

Park, S. H., Heo, N. Y., Park, J. H., Kim, T. O., Yang, S. Y., Moon, Y. S., ... Lee, H. Y. (2012). Obesity, insulin resistance, and the risk of an elevated alanine aminotransferase activity in the Korean adolescent population. Journal of Pediatric Endocrinology and Metabolism, 25, 945-949.

Pastucha, D., Filipčíková, R., Horáková, D., Radová, L., Marinov, Z., Malinčíková, J., ... Dobiáš, M. (2013). The incidence of metabolic syndrome in obese Czech children: The importance of early detection of insulin resistance using homeostatic indexes HOMAIR and QUICKI. Physiological Research, 62, 277-283.

Reaven, G. (2004). The metabolic syndrome or the insulin resistance syndrome? Different names, different concepts, and different goals. Endocrinology and Metabolism Clinics of North America, 33, 283-303.

Rodrigues, A. N., Abreu, G. R., Resende, R. S., Goncalves, W. L., \& Gouvea, S. A. (2013). Cardiovascular risk factor investigation: A pediatric issue. International Journal of General Medicine, 6, $57-66$.

Rogero Blanco, M. E., Albanil Ballesteros, M. R., Sanchez Martin, M., Rabanal Basalo, A., Olivas Dominguez, A., \& Garcia Lacalle, C. (2012). Prevalence of insulin resistance in a young adult population. Relationship with weight status. Endocrinologia y Nutrición, 59, 98-104.

Safavi, S. M., Ziaei, R., \& Maracy, M. R. (2012). Association of serum ceruloplasmin level with obesity: Some components of metabolic syndrome and high-sensitive C-reactive protein in Iran. Journal of Obesity, 2012, 951093.

Siri, W. E. (1961). Body composition from fluid spaces and density: Analysis of methods. In J. Brozeck \& A. Henschel (Eds.), Techniques for measuring body composition (pp. 223-224). Washington, DC: National Academy of Sciences, National Research Council. 
Van der Aa, M. P., Fazeli Farsani, S., Knibbe, C. A., De Boer, A., \& Van der Vorst, M. M. (2015). Population-based studies on the epidemiology of insulin resistance in children. Journal of Diabetes Research, 2015, 362-375.

Wärnberg, J., Nova, E., Moreno, L. A., Romeo, J., Mesana, M. I., \& Ruiz, J. R., ... AVENA Study Group. (2006). Inflammatory proteins are related to total and abdominal adiposity in a healthy adolescent population: The AVENA Study. American Journal of Clinical Nutrition, 84, 505-512.

Weiss, R., Dziura, J., Burgert, T. S., Tamborlane, W. V., Taksali, S. E., Yeckel, C. W., ... Caprio, S. (2004). Obesity and the metabolic syndrome in children and adolescents. New England Journal of Medicine, 350, 2362-2374.

Zimmet, P., Alberti, K. G., Kaufman, F., Tajima, N., Silink, M., \& Arslanian, S., ... International Diabetes Federation Consensus Group. (2007). The metabolic syndrome in children and adolescents: An IDF consensus report. Pediatric Diabetes, 8, 299-306

Zulet, M. A., Puchau, B., Navarro, C., Marti, A., \& Martinez, J. A. (2007). Biomarcadores del estado inflamatorio: Nexo de unión con la obesidad y complicaciones asociadas. Nutrición Hospitalaria, $22,511-527$. 